\title{
Gas cooling within the diffuse ISM of late-type galaxies
}

\author{
D. Pierini ${ }^{1,2}$, J. Lequeux ${ }^{3}$, A. Boselli ${ }^{4}$, K. J. Leech ${ }^{5}$, and H. J. Völk ${ }^{2}$ \\ 1 Ritter Astrophysical Research Center, The University of Toledo, 2801 West Bancroft, Toledo, OH 43606, USA \\ e-mail: pierini@ancona.astro.utoledo.edu \\ 2 Max-Planck-Institut für Kernphysik, Saupfercheckweg 1, 69117 Heidelberg, Germany \\ e-mail: Heinrich.Voelk@mpi-hd.mpg.de \\ 3 DEMIRM, Observatoire de Paris, 61 Av. de l'Observatoire, 75014 Paris, France \\ e-mail: James.Lequeux@obspm.fr \\ 4 Laboratoire d'Astronomie Spatiale, BP 8, Traverse du Syphon, 13376 Marseille, France \\ e-mail: Alessandro.Boselli@astrsp-mrs.fr \\ 5 ISO Data Center, Astrophysics Division, ESA Space Science Dept., PO Box 50727, \\ 28080 Madrid, Spain \\ e-mail: kleech@iso.vilspa.esa.es
}

Received 24 January 2000 / Accepted 26 April 2001

\begin{abstract}
We combine observations of spiral galaxies in the [CII] line at $158 \mu \mathrm{m}$, made with the Long Wavelength Spectrometer aboard ISO, with previous data from the Kuiper Airborne Observatory to study the origin of this line, which is the main coolant of the interstellar medium at relatively low temperatures. We also use HI and $\mathrm{CO}(1-0)$ observations of these galaxies and estimate the respective line fluxes in the same beam as the [CII] observations. We confirm the existence of a linear relation between the [CII] line intensity and the $\mathrm{CO}(1-0)$ line intensity, that we extend to intrinsically fainter galaxies. The dispersion around this relation is significant and due to variations in the far-UV flux, thus in the star formation rate. We find that for the least active galaxies of our sample, in terms of star formation, the rate of [CII] line emission per interstellar hydrogen atom is similar to that in the Solar neighbourhood. For those galaxies, most of the [CII] line emission comes probably from the diffuse cold atomic medium. In more active galaxies, considered globally, the average [CII] line emission is dominated by dense photodissociation regions and to some extent by the warm ionized diffuse medium. This is true in the central regions of many spiral galaxies, and probably even in the interarm regions of the most actively star-forming ones.
\end{abstract}

Key words. infrared: ISM: lines and bands - infrared: galaxies - galaxies: spiral - galaxies: ISM - ISM: molecules

\section{Introduction}

The $[\mathrm{CII}]\left({ }^{2} \mathrm{P}_{3 / 2}-{ }^{2} \mathrm{P}_{1 / 2}\right)(\lambda=157.7409 \mu \mathrm{m})$ line of singly ionized carbon is the dominant cooling line in the diffuse Interstellar Medium (ISM) at gas temperatures $T \leq 8 \times 10^{3} \mathrm{~K}$ (Dalgarno \& McCray 1972; Tielens \& Hollenbach 1985; Wolfire et al. 1995). Excitation of the ${ }^{2} \mathrm{P}_{3 / 2}$ level of $\mathrm{C}^{+}$is due to inelastic collisions either with neutral hydrogen atoms and molecules or with electrons (Dalgarno \& McCray 1972; Stacey 1985; Kulkarni \& Heiles 1987). Neutrals dominate over electrons if the fractional ionization $X_{\mathrm{e}}=n_{\mathrm{e}} /\left(n_{\mathrm{H}}+2 n_{\mathrm{H}_{2}}\right)$ is smaller than $10^{-3}$ (Dalgarno \& McCray 1972).

\section{Send offprint requests to: D. Pierini}

* Based on observations with the Infrared Space Observatory (ISO), an ESA project with instruments funded by ESA member states (especially the PI countries: France, Germany, The Netherlands and the UK) and with the participation of ISAS and NASA.
Heating of the interstellar gas is mainly due to photoelectrons emitted by 3-D dust grains and Polycyclic Aromatic Hydrocarbons (PAHs) submitted to ultraviolet radiation from stars (Bakes \& Tielens 1994), both in the diffuse ISM (Wolfire et al. 1995) and in Photodissociation Regions (PDRs), at the interfaces between molecular clouds and HII regions (Tielens \& Hollenbach 1985; Bakes \& Tielens 1998). The photoelectric effect is due essentially to photons with $6 \leq h \nu<13.6 \mathrm{eV}$. In the field of a galaxy, this radiation is dominated by B3 to B0 stars with $5 \leq M \leq 20 M_{\odot}$ (e.g. Xu et al. 1994) but of course hotter, more massive stars can also contribute locally.

Earlier studies of the inner regions of gas-rich spirals and starburst galaxies (Crawford et al. 1985; Stacey et al. 1991; Carral et al. 1994) have found that the [CII] line intensity, $I_{\mathrm{CII}}$, is typically a few $\times 10^{-3}$ of the total far-IR continuum dust emission, $I_{\mathrm{FIR}}$, within the same region. For these galaxies, the previous authors have also found a linear relation between $I_{\mathrm{CII}}$ and the ${ }^{12} \mathrm{CO}(\mathrm{J}: 1 \rightarrow 0)$ 
$(\lambda=2.6 \mathrm{~mm})$ line intensity, $I_{\mathrm{CO}}$, with a scatter slightly larger than one order of magnitude. Conversely, the correlation with atomic hydrogen is poor. This led them to the conclusion that PDRs are the main sources of the [CII] emission on a galaxy scale (see also Wolfire et al. 1989).

However space-resolved [CII] observations of the large spiral galaxy NGC 6946 by Madden et al. (1993) showed that this emission originates also in the diffuse ISM (mostly associated with the interarm regions). More precisely, $5 \%$ of the total line luminosity in this galaxy comes from its nucleus, $20 \%$ from the spiral arms with their HII regions and PDRs, and 75\% from an extended component, the fractional contribution of which increases with radius. This result is reproduced by the model calculations of Sauty et al. (1998).

Unfortunately, resolved galaxies on which such a detailed study can be conducted are not numerous. We will thus present a discussion of the relative contribution of the diffuse medium and of dense PDRs in spiral galaxies based on a relatively large sample of galaxies observed in the [CII] line at their central position, which add to the previous galaxies observed with the Kuiper Airborne Observatory (KAO).

A substantial number of galaxies have been observed in the [CII] line with the Long Wavelength Spectrometer (LWS) (Clegg et al. 1996) aboard ISO (Kessler et al. 1996). Malhotra et al. (1997) observed 30 normal starforming galaxies, but unfortunately do not give data for individual galaxies. Smith \& Madden (1997) report results on 5 low blue luminosity spirals in the Virgo cluster, Braine \& Hughes (1999) measurements of the normal spiral NGC 4414 and Lord et al. (1996) observations of the peculiar galaxy NGC 5713. Leech et al. (1999) detected 14 out of 19 Virgo cluster spiral galaxies with relatively low massive star-formation (SF) activity. All the latter galaxies are in general less active in terms of star-formation than the galaxies observed with the KAO.

Using the sample of Leech et al. (1999) together with the KAO sample, Pierini et al. (1999-hereafter referred to as P99) have shown that the [CII]-to-CO line intensity ratio, $I_{\mathrm{CII}} / I_{\mathrm{CO}}$, is a reliable tracer of the global massnormalized massive star-formation rate (SFR) of normal spiral galaxies, as measured by the equivalent width of the $\mathrm{H} \alpha$ line $(\mathrm{H} \alpha E W)$, which is proportional to the ratio of the stellar Lyman continuum to the stellar red continuum.

They also observed a non-linear dependence of $I_{\mathrm{CII}} / I_{\mathrm{FIR}}$ on the $\mathrm{H} \alpha E W$, where $I_{\mathrm{FIR}}$ is determined as in Helou et al. (1985). The so-called "normal star-forming" galaxies with $\mathrm{H} \alpha E W \geq 10 \AA$ have values of $I_{\mathrm{CII}} / I_{\mathrm{FIR}} \sim$ $4 \times 10^{-3}$, while the "quiescent" galaxies with $\mathrm{H} \alpha E W<$ $10 \AA$, mainly identified with early-type spirals, show a continuous decrease in $I_{\mathrm{CII}} / I_{\mathrm{FIR}}$ with decreasing $\mathrm{H} \alpha E W$. P99 interpreted the latter behaviour in terms of a dominant "[CII]-quiet" component of the far-IR emission, due to dust heating by relatively low mass stars, which do not emit enough non-ionizing far-UV radiation to produce a substantial photoelectric effect (see e.g. Xu et al. 1994). This scenario has been recently invoked to interpret the $[\mathrm{CII}]$ emission of early-type galaxies (Malhotra et al. 2000).

Assuming that the [CII] emission characteristics of diffuse and compact Galactic sources, empirically established by Nakagawa et al. (1998), are the same as those of the normal galaxies in their sample, P99 finally showed that the diffuse components of the ISM are at least as important sources of gas cooling in these galaxies as are compact regions, in agreement with recent arguments in the literature (Madden et al. 1993; Heiles 1994; Bennett et al. 1994; Dwek et al. 1997; Smith \& Madden 1997; Sauty et al. 1998).

Here we want to discuss the latter conclusion in more detail, taking into account the gas content in its molecular and atomic phases at different galactocentric radii. We will use the total sample of 42 late-type galaxies observed by Stacey et al. (1991) (the KAO sample), Smith \& Madden (1997) and Leech et al. (1999). The sample and the related data-base are discussed in Sect. 2. Our observational results are shown in Sect. 3. The discussion and the summary of our conclusions are contained in Sects. 4 and 5 , respectively.

\section{The galaxy sample and complementary data}

\subsection{The sample}

The ISO Guaranteed Time program "VIRGO" combines studies of a deep optically complete, volume-limited sample of spiral, irregular and blue compact dwarf galaxies, selected from the Virgo Cluster Catalogue (VCC) of Binggeli et al. (1985). 117 member galaxies later than $\mathrm{S} 0$ and with $B_{\mathrm{T}} \leq 18 \mathrm{mag}$ were selected in the core and at the periphery of the cluster (Boselli et al. 1997). 62 targets were observed with ISOPHOT at 60,100 and $170 \mu \mathrm{m}$ and a complete sample of 95 targets was observed with ISOCAM through the LW2 and LW3 filters centered respectively at 6.75 and $15 \mu \mathrm{m}$. Due to time constraints, only 19 normal spiral galaxies (18 with $B_{\mathrm{T}} \leq 12.3 \mathrm{mag}+\mathrm{NGC} 4491$, with $B_{\mathrm{T}}=13.4 \mathrm{mag}$ ) could be observed with the LWS in the [CII] line by Leech et al. (1999). Fourteen galaxies were detected in the [CII] line with a signal-to-noise ratio above $3 \sigma$; upper limits were set to the other 5 . Assuming a distance of $21 \mathrm{Mpc}$ from Virgo, the $70^{\prime \prime}$ half power beam-width (HPBW) of the LWS corresponds to $\sim 7 \mathrm{kpc}$ and encompasses at least one exponential disk scale-length, with the exception of the galaxies with optical major axis larger than 5 arcmin. 5 other VCC spiral galaxies with $12.5 \leq B_{\mathrm{T}} \leq 13.9$ mag, observed with the LWS in the [CII] line by Smith \& Madden (1997) are added to this sample. We call the total sample the ISO sample. This sample contains spirals with a large dynamic range in mass-normalized massive star-formation rate (SFR) $(0 \leq \mathrm{H} \alpha E W \leq 71 \AA)$ and in morphological type (from $\mathrm{S} 0 / \mathrm{a}$ to $\mathrm{Sd}$ ).

$\mathrm{H} \alpha+[\mathrm{NII}]$ equivalent widths are available for 20 out of these 24 galaxies either from CCD imaging (Boselli et al. in preparation; Gavazzi et al. in preparation) or, in a few 
Table 1. ISO sample: galaxy parameters.

\begin{tabular}{|c|c|c|c|c|c|c|c|c|}
\hline $\begin{array}{l}\text { Denomination } \\
\text { NGC / VCC }\end{array}$ & type & $\begin{array}{r}\log \mathrm{D} \\
0.1^{\prime}\end{array}$ & $\log R$ & $\begin{array}{c}\mathrm{H} \alpha \mathrm{EW} \\
\AA\end{array}$ & $\begin{array}{c}\mathrm{I}_{\mathrm{CO}} \\
\mathrm{K} \mathrm{km} \mathrm{s}{ }^{-1}\end{array}$ & $\begin{array}{c}\mathrm{N}(\mathrm{HI}) \\
10^{20} \mathrm{~cm}^{-2}\end{array}$ & $\begin{array}{c}\mathrm{F}_{\mathrm{CII}} \\
10^{-20} \mathrm{~W} \mathrm{~cm}^{-2}\end{array}$ & ref \\
\hline NGC 4178 VCC 66 & 5 & 1.71 & 0.45 & 23 & $0.60^{\mathrm{a}}$ & 33.4 & 7.80 & 12356 \\
\hline NGC 4189 VCC 89 & 5 & 1.38 & 0.14 & 20 & 3.42 & 15.9 & 9.10 & 1456 \\
\hline NGC 4192 VCC 92 & 3 & 1.99 & 0.55 & 9 & $6.24^{\mathrm{b}}$ & 9.2 & 8.80 & 1256 \\
\hline NGC $4222 \quad$ VCC 187 & 7 & 1.52 & 0.86 & 7 & 0.62 & 24.8 & 4.50 & 456 \\
\hline NGC 4293 VCC 460 & 1 & 1.75 & 0.34 & 2 & 8.43 & 3.8 & 2.70 & 26 \\
\hline NGC 4294 VCC 465 & 5 & 1.51 & 0.42 & 55 & - & 28.3 & 8.40 & 456 \\
\hline NGC 4299 VCC 491 & 6 & 1.24 & 0.03 & 74 & 0.23 & 15.7 & 6.80 & 456 \\
\hline NGC 4394 VCC 857 & 3 & 1.56 & 0.05 & 2 & $1.40^{\mathrm{c}}$ & 3.0 & 1.40 & 1256 \\
\hline NGC 4402 VCC 873 & 5 & 1.59 & 0.55 & 16 & $7.76^{\mathrm{d}}$ & 14.6 & 17.60 & 1256 \\
\hline NGC 4429 VCC 1003 & 0 & 1.75 & 0.34 & - & - & - & 3.8 & \\
\hline NGC 4438 VCC 1043 & 3 & 1.93 & 0.43 & 6 & $4.35^{\mathrm{e}}$ & - & 8.20 & 126 \\
\hline NGC 4450 VCC 1110 & 2 & 1.72 & 0.13 & 2 & $2.60^{\mathrm{f}}$ & 1.3 & 2.10 & 1236 \\
\hline NGC 4461 VCC 1158 & 1 & 1.55 & 0.39 & - & - & - & $<2.10$ & \\
\hline NGC 4477 VCC 1253 & 0 & 1.58 & 0.04 & - & - & - & $<1.60$ & \\
\hline NGC 4491 VCC 1326 & 1 & 1.23 & 0.30 & 0 & 0.96 & - & 1.30 & 3 \\
\hline NGC 4503 VCC 1412 & 1 & 1.55 & 0.33 & 2 & - & - & $<1.30$ & \\
\hline NGC 4522 VCC 1516 & 5 & 1.57 & 0.57 & 10 & 3.82 & 5.4 & 8.10 & 46 \\
\hline NGC 4569 VCC 1690 & 2 & 1.98 & 0.34 & 2 & $19.72^{\mathrm{g}}$ & 2.9 & 15.10 & 12356 \\
\hline NGC 4579 VCC 1727 & 2 & 1.77 & 0.10 & 4 & $4.68^{\mathrm{h}}$ & 4.0 & 7.80 & 1256 \\
\hline NGC 4596 VCC 1813 & 1 & 1.60 & 0.13 & 0 & $<1.54$ & - & $<2.00$ & 3 \\
\hline NGC 4608 VCC 1869 & 0 & 1.51 & 0.08 & - & - & - & $<0.60$ & \\
\hline NGC 4647 VCC 1972 & 5 & 1.46 & 0.10 & 16 & $7.99^{\mathrm{i}}$ & 7.1 & 19.00 & 1256 \\
\hline NGC 4654 VCC 1987 & 5 & 1.69 & 0.24 & 30 & $6.66^{1}$ & 10.0 & 28.40 & 1256 \\
\hline NGC 4698 VCC 2070 & 1 & 1.60 & 0.21 & 6 & $<0.99$ & 1.1 & 1.30 & 12356 \\
\hline
\end{tabular}

${ }^{\mathrm{a}}$ Point-like, ${ }^{\mathrm{b}} \mathrm{CO}$ FWHM: $125^{\prime \prime}$, ${ }^{\mathrm{c}}$ point-like, ${ }^{\mathrm{d}} \mathrm{CO}$ FWHM: $86{ }^{\prime \prime},{ }^{\mathrm{e}} \mathrm{CO}$ FWHM: $177^{\prime \prime},{ }^{\mathrm{f}}$ point-like, ${ }^{\mathrm{g}} \mathrm{CO}$ FWHM: $92^{\prime \prime}$, ${ }^{\mathrm{h}} \mathrm{CO}$ FWHM: $55^{\prime \prime},{ }^{\mathrm{i}} \mathrm{CO}$ FWHM: $137^{\prime \prime}$, ${ }^{1}$ extended, ${ }^{1}$ Stark et al. (1986), ${ }^{2}$ Kenney \& Young (1988), ${ }^{3}$ Boselli et al. (1995), ${ }^{4}$ Smith \& Madden (1997), ${ }^{5}$ Warmels (1988), ${ }^{6}$ de Vaucouleurs et al. (1991 - RC3).

cases, from long-slit spectroscopy of their central regions (Kennicutt \& Kent 1983). The latter equivalent widths represent fairly well the whole mass-normalized massive star-formation rate, since the $3^{\prime}-7^{\prime}$ slits used by Kennicutt \& Kent (1983) cover almost the entire galaxy. 19/24 galaxies have detections of the $\mathrm{CO}(1-0)$ line at their central position or upper limits. $13 / 24$ galaxies have $\mathrm{HI}(\lambda=21 \mathrm{~cm})$ surface density profiles deprojected onto the galaxy plane from Warmels (1988), while 17/24 have observed HI fluxes listed in de Vaucouleurs et al. (1991-RC3).

The parameters of the ISO sample are given in Table 1 as follows:

Column 1: NGC and VCC numbers;

Column 2: morphological type (RC3 number);

Column 3: logarithm of the length of major axis from the $\mathrm{RC} 3$;

Column 4: logarithm of the axial ratio from the RC3;

Column 5: $\mathrm{H} \alpha+[\mathrm{NII}]$ equivalent width ( $\mathrm{H} \alpha E W$ for short); Column 6: $\mathrm{CO}(1-0)$ line intensity (in the main beam temperature scale) at the central position of the galaxy, interpolated/extrapolated at/to the $70^{\prime \prime}$ LWS resolution as described in Sect. 2.2;

Column 7: average neutral hydrogen column density in the $70^{\prime \prime}$ LWS beam, $N(\mathrm{HI})$, derived as shown in Sect. 2.2; Column 8: observed [CII] line flux;
Column 9: references for the $\mathrm{CO}(1-0)$ and $\mathrm{HI}$ data. In the footnotes, we give the FWHM of the CO distribution of individual galaxies, as determined in Sect. 2.2.

Data for the KAO sample from Stacey et al. (1991 hereafter referred to as S91) are given in Table 2. These authors give average [CII] line surface brightnesses for the central regions of 18 galaxies of their sample, observed with a beam of $55^{\prime \prime}$ FWHM, from which we derive line fluxes comparable to those of Table 1.

S91 also report central measurements of the $\mathrm{CO}(1-0)$ line emission (roughly at the same angular resolution as their KAO measurements) for the whole sample and measurements in the HI line emission (at different resolutions) for 16/18 galaxies. HI flux measurements are listed in the RC3 for $17 / 18$ objects. Unfortunately, $\mathrm{H} \alpha E W$ s are available only for a third of the KAO sample, from the $3^{\prime}$ slit spectroscopy of Kennicutt \& Kent (1983) and, therefore, they are poor approximation to the integrated value of the mass-normalized massive star-formation rate.

In Table 2 we give the parameters of the KAO sample galaxies as follows:

Column 1: galaxy name;

Column 2: morphological type from the RC3;

Column 3: logarithm of the length of major axis from the $\mathrm{RC} 3$; 
Table 2. KAO sample: galaxy parameters.

\begin{tabular}{|c|c|c|c|c|c|c|c|c|}
\hline Denomination & type & $\begin{array}{r}\log \mathrm{D} \\
0.1^{\prime}\end{array}$ & $\log R$ & $\begin{array}{c}\mathrm{H} \alpha \mathrm{EW} \\
\AA\end{array}$ & $\begin{array}{c}\mathrm{I}_{\mathrm{CO}} \\
\mathrm{K} \mathrm{km} \mathrm{s}^{-1}\end{array}$ & $\begin{array}{c}\mathrm{N}(\mathrm{HI}) \\
10^{20} \mathrm{~cm}^{-2}\end{array}$ & $10^{-20} \mathrm{~W} \mathrm{~cm}^{-2}$ & flag \\
\hline NGC 660 & 1 & 1.91 & 0.41 & - & 65.69 & 26.5 & $<33.5$ & gr \\
\hline NGC 891 & 3 & 2.13 & 0.73 & - & 63.69 & 24.2 & 86.0 & gr \\
\hline Maffei 2 & 2 & 1.76 & 0.57 & - & 50.92 & 134.6 & 16.4 & $\mathrm{sb}$ \\
\hline NGC 2146 & 2 & 1.78 & 0.25 & 52 & 52.31 & 15.1 & 335.4 & $\mathrm{sb}$ \\
\hline M 82 & 3 & 2.05 & 0.41 & - & 261.54 & 19.6 & 1419.0 & $\mathrm{sb}$ \\
\hline NGC 3079 & 5 & 1.90 & 0.75 & 8 & 71.07 & 41.3 & 97.2 & gr \\
\hline NGC 3109 & 9 & 2.28 & 0.71 & - & 10.77 & 75.6 & $<29.2$ & gr \\
\hline NGC 3628 & 3 & 2.17 & 0.69 & - & 62.15 & 24.2 & 67.9 & $\mathrm{gr}$ \\
\hline NGC 3690 & 9 & 1.08 & 0.08 & - & 13.85 & - & 80.8 & $\mathrm{sb}$ \\
\hline NGC 4565 & 3 & 2.20 & 0.93 & - & 10.61 & 37.3 & 25.8 & gr \\
\hline NGC 4736 & 2 & 2.05 & 0.09 & 8 & 31.08 & 3.8 & 62.8 & gr \\
\hline NGC 5907 & 5 & 2.11 & 0.96 & - & 24.61 & 51.5 & 51.6 & gr \\
\hline NGC 6240 & 90 & 1.32 & 0.28 & - & 13.85 & 6.7 & 28.4 & $\mathrm{gr}$ \\
\hline NGC 6946 & 6 & 2.06 & 0.07 & 29 & 80.61 & 33.1 & 65.3 & gr \\
\hline NGC 1068 & 2 & 1.85 & 0.07 & 50 & 86.92 & 3.3 & 369.8 & $\mathrm{sb}$ \\
\hline IC 342 & 6 & 2.33 & 0.01 & 66 & 58.00 & 45.6 & 258.0 & $\mathrm{sb}$ \\
\hline M 51 & 4 & 2.05 & 0.21 & - & 48.92 & 12.1 & 120.4 & gr \\
\hline M 83 & 5 & 2.11 & 0.05 & - & 74.00 & 39.5 & 404.2 & $\mathrm{sb}$ \\
\hline
\end{tabular}

Column 4: logarithm of the axial ratio from the RC3; Column 5: $\mathrm{H} \alpha+[\mathrm{NII}]$ equivalent width ( $\mathrm{H} \alpha E W$ for short); Column 6: $\mathrm{CO}(1-0)$ line intensity (in the main beam temperature scale) at the central position of the galaxy, approximately at the $55^{\prime \prime} \mathrm{KAO}$ resolution;

Column 7: average neutral hydrogen column density in the $55^{\prime \prime}$ KAO beam, $N(\mathrm{HI})$, derived as shown in Sect. 2.2;

Column 8: observed [CII] line flux;

Column 9: "sb" and "gr" refer to starburst and gas-rich galaxies, respectively, i.e. to galaxies with dust temperatures in excess of $40 \mathrm{~K}(\mathrm{sb})$ and less than $40 \mathrm{~K}(\mathrm{gr})$, according to S91.

We divide the total ISO sample into quiescent and normal star-forming galaxies according to the values of $\mathrm{H} \alpha$ $E W<10 \AA$ and $\geq 10 \AA$, respectively, when $\mathrm{H} \alpha$ measurements are available.

\subsection{Data analysis}

A major difficulty in our analysis is the lack of homogeneous [CII], $\mathrm{CO}$ and $\mathrm{HI}$ data obtained at the same angular resolution. We will try to remedy this situation by interpolating/extrapolating the $\mathrm{CO}$ and $\mathrm{HI}$ measurements at/to the resolution of the [CII] observations, and will consider mainly surface brightnesses in the KAO or LWS beam.

While S91 give CO line fluxes obtained at angular resolutions rather similar to their beam, the situation is more heterogeneous for the ISO sample. CO line fluxes of Virgo galaxies have been measured at different angular resolutions by Young et al. (1985), Stark et al. (1986), Kenney \& Young (1988), Boselli et al. (1995) and Smith \& Madden (1997). Fortunately, the surveys of Stark et al. (1986) (100" HPBW) and Kenney \& Young (1988) (45" HPBW) have 28 objects in common, 11 of which belong to our ISO sample.

In Fig. 1 we reproduce the plot of the central CO line intensity (in $\mathrm{K} \mathrm{km} \mathrm{s}^{-1}$ in the main beam temperature scale) for these 28 objects as observed by these two radiotelescopes, from Kenney \& Young (1988). Detections and upper limits are considered. The filled circles represent the $11 / 28$ galaxies in common with the present study. We see that the representative points scatter nicely between a line of slope 1 expected for galaxies with a $\mathrm{CO}$ distribution much more extended than both beams, and a line of slope $(45 / 100)^{2}$ expected for distributions much smaller than both beams. In particular, it is inferred that the CO distribution of NGC 4654 is extended, while the ones of NGC 4178, NGC 4394 and NGC 4450 are pointlike. Therefore, for NGC 4654, the average CO brightness in the $70^{\prime \prime}$ ISO-LWS beam is derived from the average of the measurements at $45^{\prime \prime}$ and $100^{\prime \prime}$ resolution. Instead, for NGC 4178, NGC 4394 and NGC 4450, the average CO brightness in the ISO-LWS beam is derived by dividing the CO line flux by the ISO-LWS beam area.

For the remaining 6 ISO galaxies observed and detected in $\mathrm{CO}$ at these two different angular resolutions, we apply a different method. Assuming that both antenna beams are circular and Gaussian and that the CO brightness is Gaussian with the same shape as the optical galaxy (given by the RC3), we can derive first the FWHM of the $\mathrm{CO}$ distribution along the major axis from the observed flux ratio, then the average CO brightness in the ISO-LWS beam (also assumed to be circular Gaussian). The values of the FWHM of the $\mathrm{CO}$ distribution along the major axis of these 6 galaxies are given in the footnotes of Table 1.

For the galaxies NGC 4178, NGC 4450 and NGC 4569 the data of Boselli et al. (1995) (34 and 43" HPBW) are 


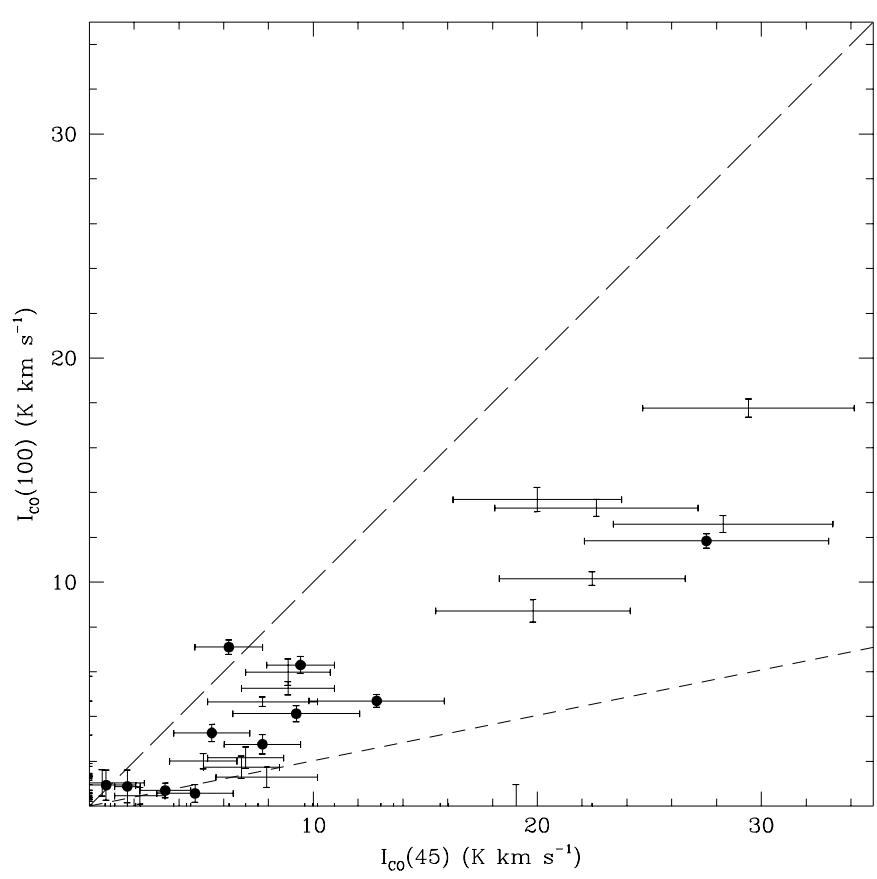

Fig. 1. Comparison of the observed central $\mathrm{CO}(1-0)$ line intensities (in $\mathrm{K} \mathrm{km} \mathrm{s}^{-1}$ in the main beam temperature scale), for 28 VCC galaxies observed by Stark et al. (1986) (100" HPBW) and by Kenney \& Young (1988) (45" HPBW), $I_{\mathrm{CO}}(100)$ and $I_{\mathrm{CO}}(45)$, respectively. Both $3 \sigma$ detections and upper limits are shown. Here, the long- and short-dashed lines reproduce the relationships between the couples of measurements in case of point-like and extended $\mathrm{CO}$ distributions with respect to the previous two beam-sizes, respectively. The filled circles represent the 11 galaxies in common with the present study.

preferred to those of Kenney \& Young (1988) since their accuracy is much better.

For the galaxy NGC 4698 only upper limits are available, so that we estimate a mean upper limit to its average CO brightness in the LWS beam.

For the remaining 7 ISO galaxies with available $\mathrm{CO}$ measurements, but taken only at one radiotelescope, we assume that the ratio of the $\mathrm{CO}$ to the optical size is the same as the average one determined from the $27 / 28$ galaxies observed by both Stark et al. (1986) and Kenney \& Young (1988) with available measurements/upper limits, and calculate the average CO brightness in the LWS beam. This method introduces some uncertainty since the average ratio of the $\mathrm{CO}$ to the optical size (0.32) has a rms dispersion of 0.30 for the 27 galaxies. We find that this ratio does not depend on the morphological type of the galaxy. The previous 7 ISO galaxies represent $40 \%$ of the ISO galaxies with estimated average CO brightness in the LWS beam and $20 \%$ of the total sample galaxies in Fig. 4.

The HI data-set is more heterogeneous. S91 report average HI column densities from maps obtained at very different angular resolutions with respect to the KAO beam. However, 17/18 galaxies of the latter sample have observed HI fluxes listed in the RC3. Although the spatial information is not available, the latter measurements constitute at least a homogeneous data-set and are preferred to those adopted by $\mathrm{S} 91$.

$17 / 24$ galaxies of the ISO sample have observed HI fluxes listed in the RC3 as well. In addition, 14 galaxies of the ISO sample have HI surface density profiles determined by Warmels (1988) after correction for the inclination of the galaxy. For these 14 ISO galaxies, we use these data to calculate the average $\mathrm{HI}$ column density $N(\mathrm{HI})$ in the $70^{\prime \prime}$ LWS beam by correcting backwards the Warmel's data into apparent surface densities, not deprojected.

For the 2 ISO galaxies (NGC 4293 and NGC 4522) and the $17 \mathrm{KAO}$ galaxies with only a HI flux, we derive a "hybrid" average surface brightness in the LWS or KAO beam by assuming that the distribution of $\mathrm{HI}$ is Gaussian with a FWHM equal to the blue light dimension as given in the RC3. Again, our assumption is reasonable but introduces some uncertainty. Such "hybrid" surface brightnesses are admittedly crude but there is no alternative.

For the 14 ISO galaxies with data from Warmels, the average ratio between the average column densities in the ISO-LWS beam derived from the HI surface brightness profiles and those estimated through the "hybrid" surface brightness method is 1.5, with a rms dispersion of 0.9 . The difference in these average surface brightnesses is not large enough to justify a further correction. Although the dispersion is large, we are confident that the conclusions derived in the following sections are not biased by the use of these crude HI surface brightnesses.

Note that in all the previous discussion we have eliminated the interacting galaxy NGC 4438, which is a special case.

\section{Results}

Due to our observational limitations in terms of angular resolution, we find useful to quantify the fraction of a galaxy observed either with the KAO or with the ISOLWS in terms of a coverage factor $C F$. This coverage factor is the ratio between the area of the observing beam and the projected optical galaxy area as defined in the RC3.

Figure 2 shows a plot of the observed central [CII] line intensity vs. the $C F$ of the 42 galaxies of our sample. It confirms that the KAO probed mainly the innermost regions of both gas-rich and starburst galaxies. This is in part because the galaxies of this sample are relatively nearby. The ISO galaxies are further away and as a consequence the LWS sampled larger areas of the galaxies. We note that the 5 ISO galaxies with [CII] line upper limits fall well within the range of values of $C F$ of the galaxies detected with the LWS, so that we are led to the conclusion that their faintness is not due to a particularly reduced spatial sampling but to a genuine deficiency of [CII] line emission. The Hubble type of these 5 undetected galaxies is either $\mathrm{S} 0 /$ a or Sa; galaxies of these types have generally a lower SFR than galaxies of later type, so the faintness of their emission is not unexpected. 


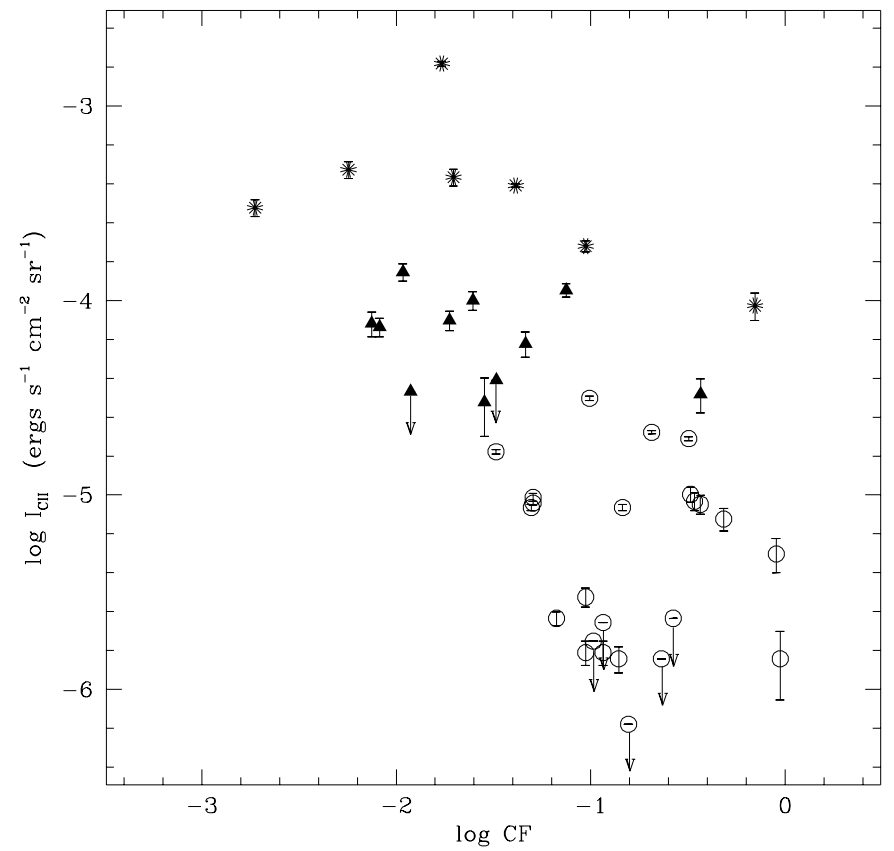

Fig. 2. The observed central $[\mathrm{CII}]$ line intensity, $I_{\mathrm{CII}}$, vs. the coverage factor, $C F$, defined as the ratio between the area of the observing beam and the projected optical galaxy area as defined in the RC3. Hereafter, asterisks and filled triangles denote starburst galaxies and gas-rich galaxies of the KAO sample, respectively, while open circles identify spiral galaxies of the ISO sample (see text). Arrows define upper limits.

In Fig. 3, we plot as a function of the $C F$ the average column densities of molecular hydrogen in the beam, $2 \mathrm{~N}\left(\mathrm{H}_{2}\right)$, (in terms of $\mathrm{HI}$ nuclei) and those of the neutral atomic hydrogen, also in the beam, $N(\mathrm{HI})$, both not deprojected from the inclination of the galaxy (panels a and b, respectively). $2 N\left(\mathrm{H}_{2}\right)$ is derived from $I_{\mathrm{CO}}$ as follows (Digel et al. 1995):

$2 N\left(\mathrm{H}_{2}\right)=2.12( \pm 0.28) \times 10^{20} I_{\mathrm{CO}}$,

where $2 N\left(\mathrm{H}_{2}\right)$ is in units of $\mathrm{cm}^{-2}$ and $I_{\mathrm{CO}}$ is in units of $\mathrm{K} \mathrm{km} \mathrm{s}^{-1}$.

This value of the $\mathrm{H}_{2}$ column density-to- $\mathrm{CO}$ line intensity ratio has been derived in Orion and we use it for all our galaxies, although it may depend on metallicity and on the ISRF intensity (cf. Israel 1997 and references therein). Its uncertainty is equivalent to $13 \%$ of $2 N\left(\mathrm{H}_{2}\right)$.

We arbitrarily assume that both $N(\mathrm{HI})$ and $2 N\left(\mathrm{H}_{2}\right)$ are affected by a $1 \sigma$ uncertainty of $20 \%$.

For both starburst and non-starburst galaxies, the inner regions are richer in molecular hydrogen than the outer regions (cf. Young \& Knezek 1989). This is not the case for atomic hydrogen. We note that the ISO early-type spiral galaxies have the lowest values of $N(\mathrm{HI})$ (cf. Tables 1 and 2), in agreement with Solanes et al. (2001).

The innermost regions of the KAO galaxies are rich in molecular gas, as witnessed by their high molecular-toatomic hydrogen column density ratio $\left(2 N\left(\mathrm{H}_{2}\right) / N(\mathrm{HI}) \geq\right.$ 4 ), or more exactly by their high $\mathrm{CO} / \mathrm{HI}$ line intensity ratio. Therefore, we might reasonably expect that, in these regions, a relevant part of the gas cooling via [CII] line

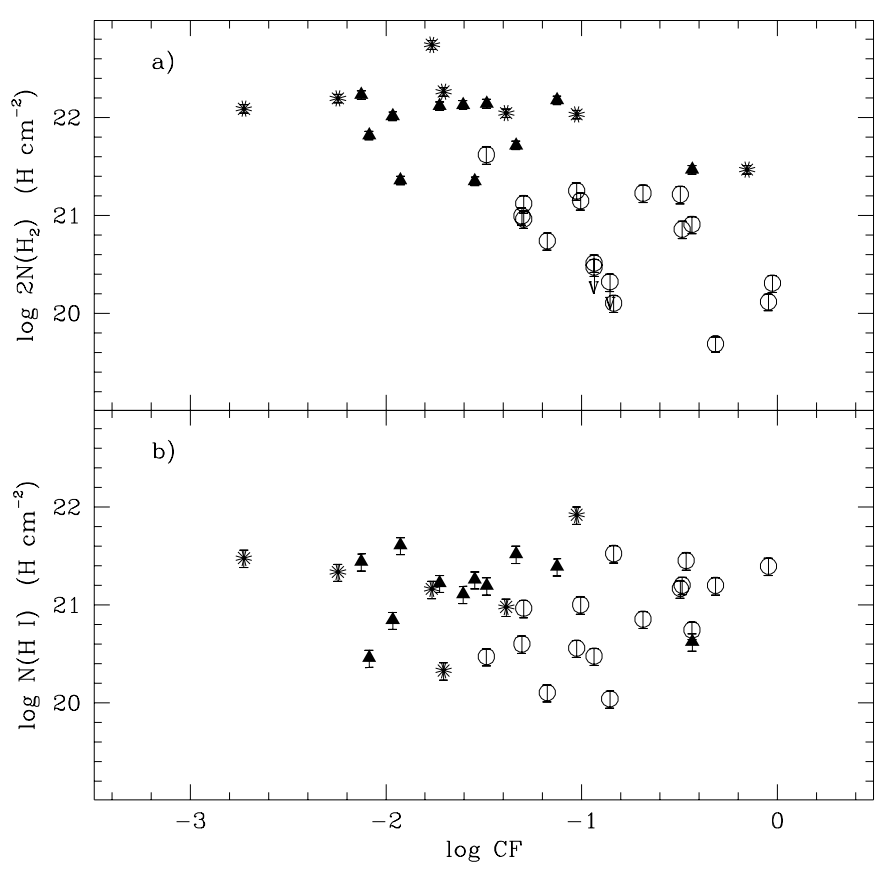

Fig. 3. The average column density of molecular hydrogen in the beam, $2 N\left(\mathrm{H}_{2}\right)$, (in terms of $\mathrm{HI}$ nuclei) a) and the average column density of neutral atomic hydrogen in the beam, $N(\mathrm{HI}), \mathbf{b})$ vs. the coverage factor $C F$. See text for the determination of $2 N\left(\mathrm{H}_{2}\right) .2 N\left(\mathrm{H}_{2}\right)$ and $N(\mathrm{HI})$ are not deprojected from the inclination of the galaxy.

emission comes from PDRs associated with molecular clouds where star-formation activity may be more or less active. In particular, this may account partly for the linear relationship between $I_{\mathrm{CII}}$ and $I_{\mathrm{CO}}$ found by $\mathrm{S} 91$ for their starburst galaxies.

In Fig. 4 we show this relationship for the 36 galaxies of our total sample that are detected at the $3 \sigma$ level in at least one of the two lines, except for NGC 4596. The long-and short-dashed lines represent the two characteristic values of $I_{\mathrm{CII}} / I_{\mathrm{CO}}$ given by S91 for their "warm dust galaxies" (i.e. starburst galaxies) and "cold dust galaxies" (i.e. normally active gas-rich galaxies), respectively. The ISO observations extend this relation to line fluxes fainter by 1.5 orders of magnitude. The overall linear relation remains, although with a probably more significant rather large scatter, even though part of it is due to the uncertainties of our data analysis (cf. Sect. 2.2). The interpretation given by $\mathrm{P} 99$ for this scatter is that $I_{\mathrm{CII}} / I_{\mathrm{CO}}$ is proportional to the strength of the far-UV radiation field, as represented by the $\mathrm{H} \alpha$ equivalent width. This is confirmed by the model calculations of PDRs of various densities and of the diffuse ISM presented by Kaufman et al. (1999), their Fig. 9.

In Fig. 4, three ISO galaxies (NGC 4178, NGC 4222 and NGC 4299) have exceptionally high values of $I_{\mathrm{CII}} / I_{\mathrm{CO}}$, typical of starburst galaxies, while their $I_{\mathrm{CII}}$ are slightly lower than for galaxies of the same Hubble type and with similar $C F$ s. NGC 4222 has a particularly high inclination but small $\mathrm{H} \alpha E W$ (cf. Table 1), so that its "anomalous" behaviour in Fig. 4 is probably spurious. 


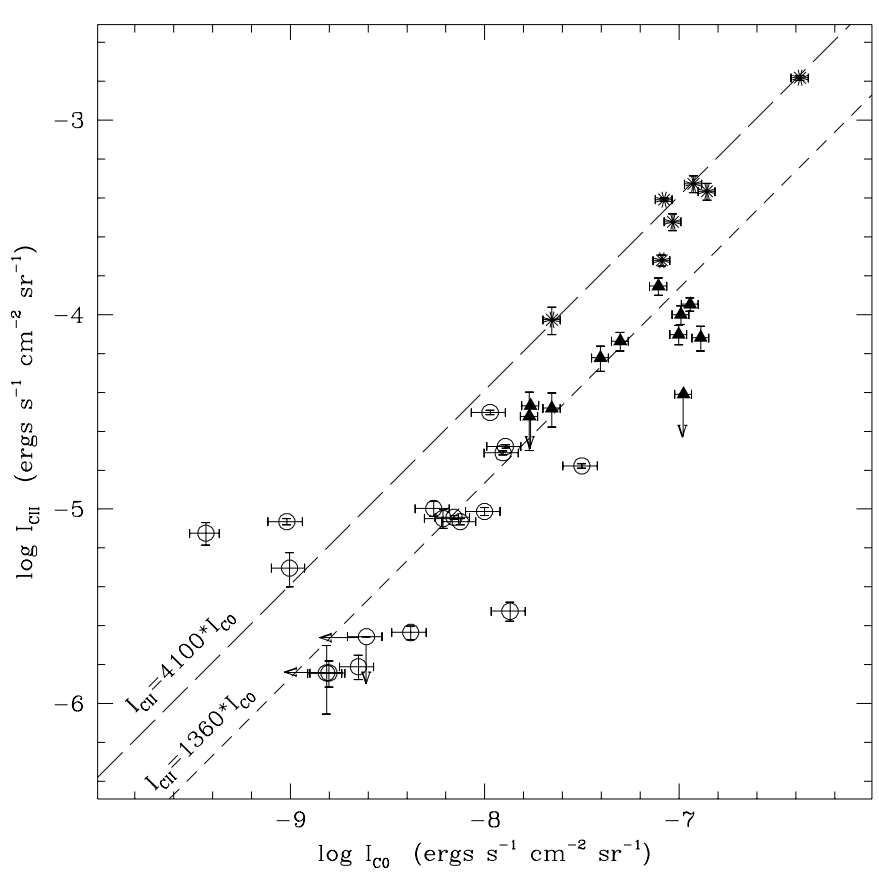

Fig. 4. The relationship between the observed central [CII] line intensity, $I_{\mathrm{CII}}$, and the central $\mathrm{CO}$ line intensity, $I_{\mathrm{CO}}$, as derived in Sect. 2.2, not deprojected from the inclination of the galaxy. Here, the long- and short-dashed lines show the average ratios of the two observables obtained by Stacey et al. (1991) for the starburst galaxies and the gas-rich galaxies, respectively.

In fact, the latter vanishes when correcting $I_{\mathrm{CII}}$ for inclination (cf. Wolfire et al. 1989), while the "anomalous" behaviours of NGC 4178 and NGC 4299 are confirmed. NGC 4178 is an HII galaxy, with some giant HII regions in its inner part (Boselli et al. in preparation), probed by LWS. Its "anomalous" behaviour was not noted by P99 due to the different value of $I_{\mathrm{CO}}$ adopted by these authors but is not due to the assumption that its CO morphology is point-like (Sect. 2.2). In fact, $I_{\mathrm{CII}} / I_{\mathrm{CO}}$ decreases by only a factor of -0.18 in logarithm under the assumption that the CO surface brightness distribution of NGC 4178 is partially resolved at $43^{\prime \prime}$ resolution. NGC 4178 and NGC 4299 have bluer $B-V$ colour indices (Gavazzi, private communication) than the other ISO galaxies of the same Hubble type considered here. In addition, they have also exceptionally low values of $2 N\left(\mathrm{H}_{2}\right) / N(\mathrm{HI})$. These behaviours lead us to the conclusion that NGC 4178 and NGC 4299 have intrinsic particularly intense ISRFs (cf. Israel 1997 and references therein) and/or intrinsic low metallicity (cf. Smith \& Madden 1997).

\section{Discussion: $[\mathrm{CII}]$ line emission by the diffuse ISM}

The correlations presented above have not yet shed light about the [CII] line emission by the diffuse ISM. Before entering this discussion, it is interesting to summarize what we know on our own Galaxy.
The diffuse ISM in our own Galaxy is thought to consist (Kulkarni \& Heiles 1987; Heiles 1988, 1994; Reynolds 1993; Heiles et al. 1996) of: i) a Cold Neutral Medium (CNM), with a temperature $T$ of about $80 \mathrm{~K}$, a hydrogen density $n_{\mathrm{H}}$ of about $90 \mathrm{~cm}^{-3}$ and a fractional ionization $X_{\mathrm{e}}$ within a factor of 3 of $\sim 6 \times 10^{-4}$; ii) a Warm Neutral Medium (WNM), with $T \sim 8000 \mathrm{~K}, n_{\mathrm{H}} \leq 1 \mathrm{~cm}^{-3}$ and $X_{\mathrm{e}} \sim 3 \times 10^{-2}$; iii) a Warm Ionized Medium (WIM), with $T \sim 8000 \mathrm{~K}, n_{\mathrm{H}} \leq 1 \mathrm{~cm}^{-3}$ and $X_{\mathrm{e}} \geq 0.75$. The distinction between WNM and WIM is not completely clear, and it may be that they are partly one and the same thing.

In our Galaxy, the high latitude [CII] line emission is associated with the CNM (Bennett et al. 1995) while, in the inner regions of the disk, the WIM has been proposed as the main source of the [CII] line emission (Heiles 1994). At high Galactic latitudes, no prominent OB associations are found, while the opposite is true for the inner regions (Bronfman et al. 2000), favouring the WNM and WIM. For the local Galaxy near the Sun, Bennett et al. (1995) find a [CII] line emission per interstellar hydrogen nucleus of $\sim 2.5 \times 10^{-26} \mathrm{ergs} \mathrm{s}^{-1} \mathrm{H}^{-1}$, while Madden et al. (1993) find a value of $\sim 1.6 \times 10^{-25} \mathrm{ergs} \mathrm{s}^{-1} \mathrm{H}^{-1}$ for the diffuse ISM of NGC 6946, an active star-forming galaxy. It is interesting to compare these observations with the results of the model calculations of Wolfire et al. (1995) which relate to the local Galactic ISM: they predict a cooling rate per hydrogen nucleus (dominated by the [CII] line) from 2.6 to $6.6 \times 10^{-26} \mathrm{ergs} \mathrm{s}^{-1} \mathrm{H}^{-1}$ in the CNM and from 0.3 to $0.8 \times 10^{-26} \mathrm{ergs} \mathrm{s}^{-1} \mathrm{H}^{-1}$ in the WNM, depending on the exact values of the physical parameters of the medium. Moreover, they estimate a value of about $\sim 2.7 \times 10^{-26}(P / k)_{3} \operatorname{ergs~s}^{-1} \mathrm{H}^{-1}$ in the WIM, where $(P / k)_{3}$ is the WIM pressure in units of $10^{3} \mathrm{~K} \mathrm{~cm}^{-3}$. Their lower value for the CNM compares favourably with the observation of Bennett et al. (1995). An increase of the UV flux would raise the cooling rate from the CNM appreciably, but this flux should be higher by considerably more than one order of magnitude to reach the cooling rate of the interarm medium of NGC 6946 (see Fig. 3 of Kaufman et al. 1999 and Table 5 of Wolfire et al. 1995).

In Fig. 5, we plot the quantity $\Lambda=4 \pi I_{\mathrm{CII}} / N(\mathrm{HI})$ for our galaxies as a function of the coverage factor $C F$. If the diffuse ISM was the only source of the [CII] line emission, this quantity should be in the same range of values than the numbers given above for the diffuse ISM of our Galaxy and most probably lower than or equal to that for the interarm medium of NGC 6946. Any contribution of the dense PDRs would raise $\Lambda$. Figure 5 shows that when the beam covers most of the galaxy $(C F \gg 0.1) \Lambda$ is indeed in this range, while it increases strongly when only the central regions are sampled. This demonstrates the importance of [CII] line emission by the diffuse ISM in nonstarburst galaxies when one considers the whole galaxy. Moreover the above discussion shows that the CNM dominates the emission in normal circumstances.

We note that the normal star-forming galaxy NGC 4178 has a relatively low value of $\Lambda$. This is not 


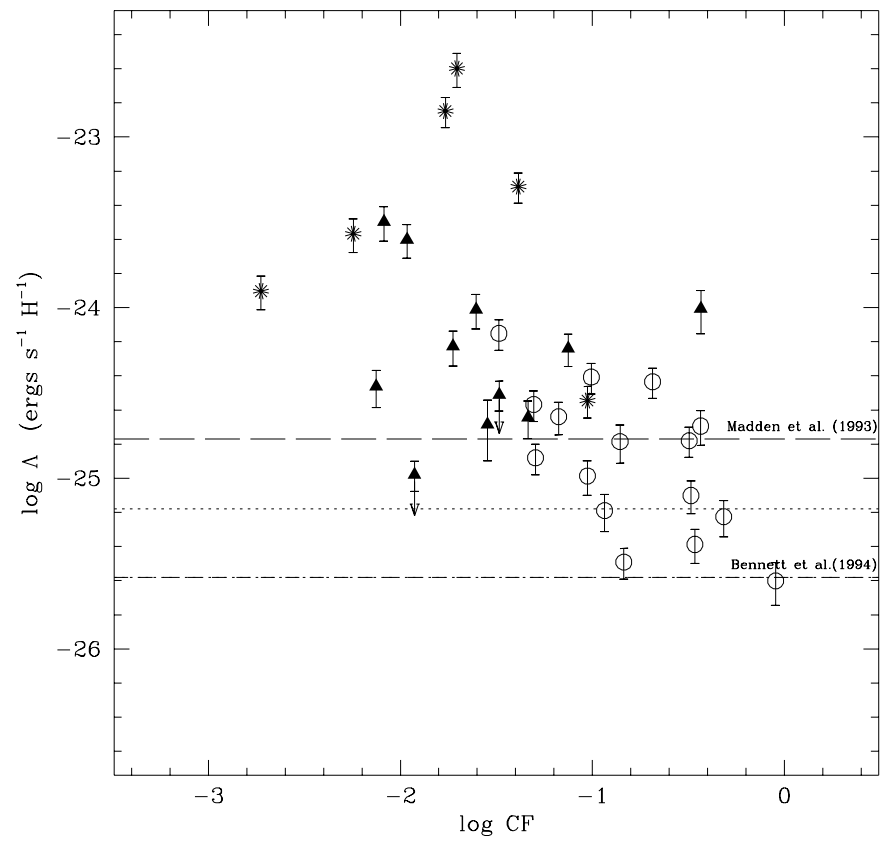

Fig. 5. The rate of $[\mathrm{CII}]$ line emission per interstellar hydrogen atom, $\Lambda=4 \pi I_{\mathrm{CII}} / N(\mathrm{HI})$, vs. the $C F$. Here, the long- and short-dashed lines show the rates of [CII] line emission per $\mathrm{H}$ atom found by Madden et al. (1993) for the diffuse ISM of the actively star-forming galaxy NGC 6946 and by Bennet et al. (1994) for the high latitude regions of our Galaxy, respectively (see text). Instead, the two dotted lines delimit the range of values of the rate of $[\mathrm{CII}]$ line emission per $\mathrm{H}$ atom in the cold neutral medium, as calculated by Wolfire et al. (1995). (N.B.: the lower dotted line is almost indistinguishable from the short-dashed line.)

unexpected if NGC 4178 is indeed a low metallicity galaxy (Wolfire et al. 1995).

We present in Fig. 6 the same plot as in Fig. 5 but considering this time the line emission per total hydrogen atom whether included or not in molecules $\left(\Lambda^{\prime}=4 \pi I_{\mathrm{CII}} /\left(N(\mathrm{HI})+2 N\left(\mathrm{H}_{2}\right)\right)\right.$. Since the contribution of $\mathrm{H}_{2}$ to the total gas is minor at the scale of a whole galaxy, both plots are very similar at large values of $C F$. This is not the case in the inner regions which correspond to small values of $C F$, but the [CII] emission per total $\mathrm{H}$ is not very much higher there than for entire galaxies. This conclusion is however uncertain because the conversion factor between the $\mathrm{CO}(1-0)$ line intensity and the column density of $\mathrm{H}_{2}$ is very poorly known in the center of galaxies. As expected, galaxies with more active SF have on average a higher value of the cooling rate per hydrogen nucleus. This confirms that the total [CII] line emission per hydrogen nucleus increases with the strength of the local far-UV field.

From our own data alone it is not directly possible to infer the fraction of the [CII] line luminosity which comes from the diffuse ISM and from the PDRs respectively, because these data are spatially averaged at our limited angular resolution. However we note that the lowest values of $\Lambda$ in Fig. 5 are similar to the local value determined by

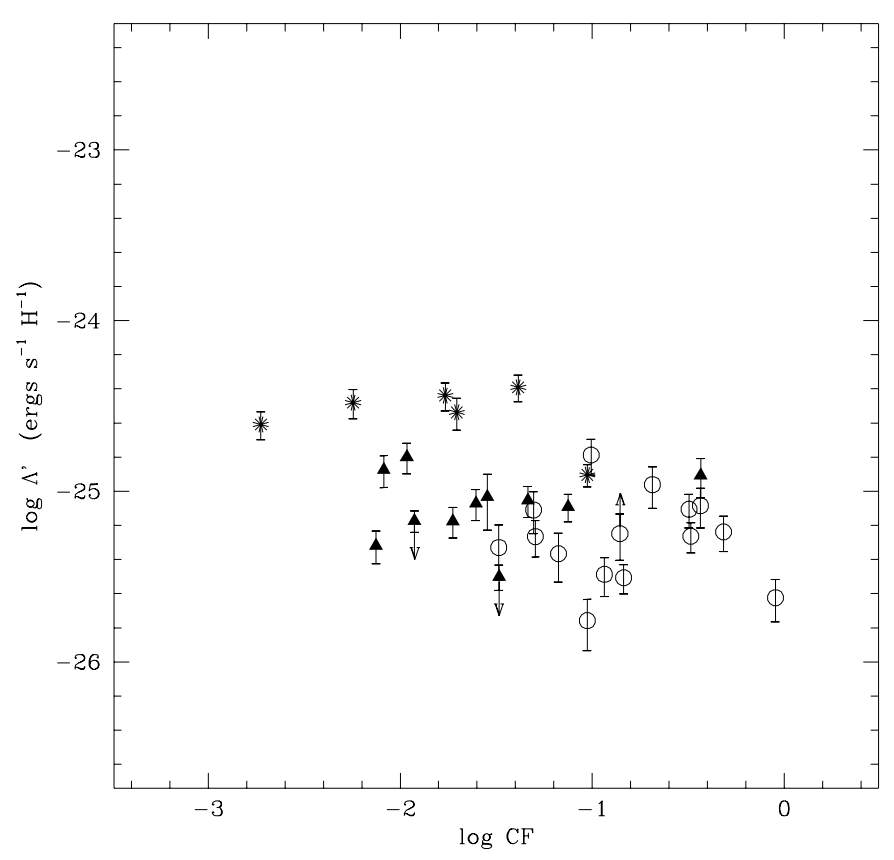

Fig. 6. The rate of $[\mathrm{CII}]$ line emission per interstellar hydrogen nucleus, $\Lambda^{\prime}=4 \pi I_{\mathrm{CII}} /\left(N(\mathrm{HI})+2 N\left(\mathrm{H}_{2}\right)\right)$, vs. the $C F$.

Bennett et al. (1995) for the solar neighbourhood. Because the [CII] line intensity is relatively insensitive to the farUV flux density, this local value should be typical for relatively quiescent galaxies. Consequently there is little room left for the contribution of PDRs to the [CII] line luminosity for the galaxies with the lowest values of $\Lambda$ in Fig. 5 . On the other hand, the high value quoted for the interarm ISM of NGC 6946 by Madden et al. (1993) would require a very high far-UV radiation density, perhaps unrealistic, if it is assumed to be due to a cold neutral medium alone, (Wolfire et al. 1995), and therefore it is likely that there is already a contribution of dense PDRs at such high values of $\Lambda$. The statistical results of P99 are consistent with this estimate. Since they used different local Galactic data and a different parametrization, our result is also complementary to P99.

In the inner regions of the sample galaxies, dense PDRs always seem to dominate as shown by the high values of $\Lambda$ in Fig. 5, even though the WIM could give a non-negligible contribution (Heiles 1994).

\section{Conclusions}

We have made or collected observations of the central regions of a relatively large sample of spiral galaxies in the $[\mathrm{CII}]\left({ }^{2} \mathrm{P}_{3 / 2}-{ }^{2} \mathrm{P}_{1 / 2}\right)(\lambda=158 \mu \mathrm{m})$ gas cooling line. This sample, mainly observed with the Long Wavelength Spectrometer aboard ISO, enlarges a previous sample observed with the Kuiper Airborne Observatory and contains on average more quiescent, fainter galaxies.

For the total KAO + ISO-LWS sample of 42 galaxies, we have collected measurements of the atomic and molecular gas, and estimated their average column densities in the same beam as the [CII] line observations. This beam 
covers various fractions of the galaxy disks according to their distance and linear size.

Our results are more detailed than but complementary to those of Pierini et al. (1999). We confirm the existence of an overall linear relation between the $[\mathrm{CII}]$ line intensity, $I_{\mathrm{CII}}$, and the $\mathrm{CO}(1-0)$ line intensity, $I_{\mathrm{CO}}$, over almost 2.5 orders of magnitude in both quantities. The significant large scatter around this relation is due to the variation of the far-UV radiation field and is in agreement with model calculations by Kaufman et al. (1999).

We find that the [CII] line emissivity per interstellar atomic hydrogen nucleus, $\Lambda$, ranges between $\sim 2.6 \times 10^{-26}$ and $\sim 4 \times 10^{-25} \mathrm{ergs} \mathrm{s}^{-1} \mathrm{H}^{-1}$ in galaxies where the observing beam covers at least $10 \%$ of the optical surface of the galaxy. The lowest observed values of $\Lambda$ are similar to the value obtained by Bennett et al. (1995) for the diffuse ISM near the Sun, where the atomic cold neutral medium (CNM) dominates the emission. For the corresponding galaxies, it is likely that most of the [CII] line emission also comes from the CNM. The dense Photodissociation Regions plus the Warm Ionized Medium dominate in more active star-forming galaxies, even including their extended regions, and they do so in the inner parts of all spiral galaxies.

Finally, we find that the cooling rate per hydrogen nucleus, when both the molecular and the atomic phases are taken into account, increases moderately with the starformation rate, although it is difficult to give a quantitative result because of the uncertainty in the amount of $\mathrm{H}_{2}$ in regions of intense star-formation.

Acknowledgements. We are grateful for the support of this work to the Deutsche Agentur für Raumfahrt Angelegenheiten, through DARA project number 50-OR-9501B.

We are indebted to G. Gavazzi for providing us with optical data prior their publication.

\section{References}

Bakes, E. L. O., \& Tielens, A. G. G. M. 1994, ApJ, 427, 822 Bakes, E. L. O., \& Tielens, A. G. G. M. 1998, ApJ, 499, 258

Bennett, C. L., Fixsen, D. J., Hinshaw, G., et al. 1994, ApJ, 434,587

Binggeli, B., Sandage, A., \& Tammann, G. A. 1985, AJ, 90, 1681 (VCC)

Boselli, A., Casoli, F., \& Lequeux, J. 1995, A\&AS, 110, 521

Boselli, A., Tuffs, R. J., Gavazzi, G., et al. 1997, A\&AS, 121, 507

Bronfman, L., Casassus, S., May, J., \& Nyman, L.- $\AA 2000$, A\&A, 358, 521

Braine, J., \& Hughes, D. H. 1999, A\&A, 344, 779

Carral, P., Hollenbach, D. J., Lord, S. D., et al. 1994, ApJ, 423, 223

Clegg, P. E., Ade, P. A. R., Armand, C., et al. 1996, A\&A, 315, L38
Crawford, M. K., Genzel, R., Townes, C. H., \& Watson, D. M. 1985, ApJ, 291, 755

Dalgarno, A., \& McCray, R. 1972, ARA\&A, 10, 375

de Vaucouleurs, G., de Vaucouleurs, A., Corwin, H. G., et al. 1991, in Third Reference Catalogue of Bright Galaxies (Springer-Verlag, New York) (RC3)

Digel, S. W., Hunter, S. D., \& Mukherjee, R. 1995, ApJ, 441, 270

Dwek, E., Arendt, R., Fixsen, D., et al. 1997, ApJ, 475, 565

Heiles, C. 1988, in The Outer Galaxy, ed. L. Blitz, \& F. J. Lockman, Lecture Notes in Physics (Springer, Berlin), 306, 151

Heiles, C. 1994, ApJ, 436, 720

Heiles, C., Reach, W. T., \& Koo, B.-C. 1996, ApJ, 466, 191

Helou, G., Soifer, B. T., \& Rowan-Robinson, M. 1985, ApJ, 298, L7

Israel, F. P. 1997, A\&A, 317, 65

Kaufman, M. J., Wolfire, M. G., Hollenbach, D. J., \& Luhman, M. L. 1999, ApJ, 527, 795

Kenney, J. D., \& Young, J. S. 1988, ApJS, 66, 261

Kennicutt, R. C., \& Kent, S. M. 1983, AJ, 88, 1094

Kessler, M. F., Steinz, J. A., Anderegg, M. E., et al. 1996, A\&A, 315, L27

Kulkarni, S., \& Heiles, C. 1987, in Interstellar Processes, ed. D. J. Hollenbach, \& H. A. Thronson (Reidel, Dordrecht), 87

Leech, K. J., Völk, H. J., Heinrichsen, I., et al. 1999, MNRAS, 310,317

Lord, S. D., Malhotra, S., Lim, T., et al. 1996, A\&A, 315, L117

Madden, S. C., Geis, N., Genzel, R., et al. 1993, ApJ, 407, 579

Malhotra, S., Helou, G., Stacey, G., et al. 1997, ApJ, 491, L27

Malhotra, S., Hollenbach, D., Helou, G., et al. 2000, ApJ, 543, 634

Nakagawa, T., Yui, Y. Y., Doi, Y., et al. 1998, ApJS, 115, 259

Pierini, D., Leech, K. J., Tuffs, R. J., \& Völk, H. J. 1999, MNRAS, 303, L29 (P99)

Reynolds, R. J. 1993, in Massive Stars: Their Lives in the Interstellar Medium, ed. J. P. Cassinelli, \& E. B. Churchwell, ASP Conf. Ser., 35, 338

Sauty, S., Gerin, M., \& Casoli, F. 1998, A\&A, 339, 19

Smith, B. J., \& Madden, S. C. 1997, AJ, 114, 138

Solanes, J. M., Manrique, A., García-Gómez, C., et al. 2001, ApJ, 548, 97

Stacey, G. J. 1985, Ph.D. Thesis, Cornell Univ.

Stacey, G. J., Geis, N., Genzel, R., et al. 1991, ApJ, 373, 423 (S91)

Stark, A. A., Knapp, G. R., Bally, J., et al. 1986, ApJ, 310, 660

Tielens, A. G. G. M., \& Hollenbach, D. 1985, ApJ, 291, 722

Warmels, R. H. 1988, A\&AS, 72, 427

Wolfire, M. G., Hollenbach, D., \& Tielens, A. G. G. M. 1989, ApJ, 344, 770

Wolfire, M. G., Hollenbach, D., McKee, C. F., et al. 1995, ApJ, 443,152

Young, J. S., Scoville, N. Z., \& Brady, E. 1985, ApJ, 288, 487

Young, J. S., \& Knezek, P. M. 1989, ApJ, 347, L35

Xu, C., Lisenfeld, U., Völk, H. J., \& Wunderlich, E. 1994, A\&A, 282,19 